\title{
Chronological changes of the amplitude-integrated EEG in a neonate with molybdenum cofactor deficiency
}

\author{
Sintha D. Sie • Rogier C. J. de Jonge $\cdot$ Henk J. Blom • \\ Margot F. Mulder • Jochen Reiss $\cdot$ R. J. Vermeulen • \\ Cacha M. P. C. D. Peeters-Scholte
}

Received: 7 May 2010 /Revised: 22 August 2010 /Accepted: 24 August 2010 /Published online: 24 September 2010

(C) The Author(s) 2010. This article is published with open access at Springerlink.com

\begin{abstract}
Molybdenum cofactor (Moco) deficiency is a rare neurometabolic disorder, characterized by neurological impairment and refractive seizures, due to toxic accumulation of sulfite in the brain. Earlier it was suggested that in Moco-deficient humans maternal clearance of neurotoxic metabolites prevents prenatal brain damage. However, limited data are available about the time profile in which neurophysiologic deterioration occurs after birth. The amplitude-integrated electroencephalography (aEEG) is a bedside method in neonates to monitor cerebral recovery after hypoxic-ischemic insults, detect epileptic activity, and evaluate antiepileptic drug treatment. We describe a chronological series of changes in aEEG tracings in a neonate with Moco deficiency. He presented with myo-
\end{abstract}

Communicated by: Georg Hoffmann

Competing interest: None declared.

S. D. Sie $(\bowtie) \cdot$ R. C. J. de Jonge

Department of Neonatology, VU University Medical Center,

P.O. Box 7057, 1007 MB Amsterdam, The Netherlands

e-mail: s.sie@vumc.nl

H. J. Blom

Department of Clinical Chemistry, VU University Medical Center, Amsterdam, The Netherlands

e-mail: h.blom@vumc.nl

\section{F. Mulder}

Department of Metabolic Disorders,

VU University Medical Center,

Amsterdam, The Netherlands

e-mail: mf.mulder@vumc.nl

\section{J. Reiss}

Institut für Humangenetik, Universitätskliniken Göttingen,

Göttingen, Germany

e-mail: jreiss@gwdg.de clonic spasms and hypertonicity a few hours after birth, however, the aEEG pattern was still normal. Within 2 days, the aEEG rapidly changed into a burst suppression pattern with repetitive seizures. After antiepileptic treatment, the aEEG remained abnormal. In this patient, the normal aEEG pattern at birth may have been due to maternal clearance of sulfite in utero. After birth, accumulation of sulfite causes progressive brain damage, reflected by the progressive depression of the aEEG tracings. This is in agreement with the results from a Moco-deficient mouse model, suggesting that maternal sulfite clearance suppresses prenatal brain damage. To our knowledge, this is the first case report describing the chronological changes in the aEEG pattern in a Moco-deficient patient. Insight into the time profile in

R. J. Vermeulen · C. M. P. C. D. Peeters-Scholte

Department of Child Neurology, VU University Medical Center, Amsterdam, The Netherlands

R. J. Vermeulen

e-mail: rj.vermeulen@vumc.nl

C. M. P. C. D. Peeters-Scholte

e-mail: c.m.p.c.d.peeters-scholte@lumc.nl

Present Address:

R. C. J. de Jonge

Department of Neonatology, Emma Children's Hospital,

Academic Medical Center,

Amsterdam, The Netherlands

e-mail: r.c.dejonge@amc.nl

Present Address:

C. M. P. C. D. Peeters-Scholte

Department of Pediatric Neurology,

Leiden University Medical Center,

Leiden, The Netherlands 
which neurologic deterioration in Moco-deficient humans occurs is essential, especially when potential treatment strategies are being evaluated.

Abbreviations
$\begin{array}{ll}\text { aEEG } & \text { Amplitude-integrated electroencephalography } \\ \text { Moco } & \text { Molybdenum cofactor } \\ \text { cPMP } & \text { Cyclic pyranopterin monophosphate } \\ \text { CSF } & \text { Cerebrospinal fluid } \\ \text { MRI } & \text { Magnetic resonance imaging }\end{array}$

\section{Introduction}

Molybdenum cofactor (Moco) deficiency is an autosomalrecessive inherited disorder leading to a combined deficiency of sulfite oxidase, xanthine dehydrogenase, and aldehyde oxidase. Deficiency of sulfite oxidase leads to toxic sulfite accumulation in the brain, causing severe neurological impairment and refractive seizures. In spite of novel antiepileptic drugs, prognosis remains poor due to progressive neurodegeneration (Johnson and Duran 2001). Currently, an effective therapy is only available for type A of the disease caused by mutations in the MOCS1 gene (Reiss and Johnson 2003).

The amplitude-integrated EEG (aEEG) is a clinical bedside system to monitor the electrocortical neuronal activity. Mostly, it is used in neonatal intensive care units for the evaluation of cerebral recovery after hypoxicischemic insults, the detection of epileptic activity, and the evaluation of antiepileptic drug treatment. Due to its simplicity a non-expert can be trained to recognize the different aEEG patterns (Hellstrom-Westas and Rosen 2006).

Results from a Moco-deficient mouse model suggest that maternal sulfite clearance suppresses prenatal brain damage (Reiss et al. 2005). Therefore, it is hypothesized that in Moco-deficient humans there is no or only moderate brain damage present at birth, but damage can develop due to progressive accumulation of toxic substances. To our knowledge, limited data are available about the time profile over which neurophysiologic deterioration occurs. In this case report we describe a chronological series of changes in aEEG tracings in a neonate with Moco deficiency.

\section{Case report}

A term male infant (39 weeks and 6 days of gestation) was delivered after an uneventful pregnancy and uncomplicated delivery with a birth weight of $3,975 \mathrm{~g}$ ( 75 th percentile). He was the fourth child of consanguineous Afghan parents. The parents and the three older siblings are all healthy.
Because of myoclonic movements a few hours after birth, he was transferred to the neonatology unit of our hospital on day 1 of life. He had mild dysmorphic features including bilateral microphthalmia, and a wide nasal bridge. On neurological examination a high pitched cry was noticed, together with hypertonicity, decreased spontaneous movements and frequent myoclonic spasms. Biochemical investigations revealed elevated levels of serum lactate $(5.7 \mathrm{mmol} / \mathrm{l})$ and creatine kinase $(7500 \mathrm{IU} / \mathrm{l})$. Serum electrolytes and glucose were all in normal range. A possible infectious cause of the symptoms was treated with intravenously administered antibiotics and acyclovir for $48 \mathrm{~h}$, until blood and cerebrospinal (CSF) fluid cultures and PCR for herpes in CSF were negative. In addition, metabolic investigation of blood and urine samples was performed, as well as blood analysis for genetic and chromosomal abnormalities. Ophthalmologic examination and cranial ultrasound were performed on admission and showed no abnormalities. A two-channel aEEG (BrainZ, Natus Medical, San Carlos, CA, USA) was started to evaluate a possible epileptic cause for the myoclonic spasms. However, a continuous normal voltage pattern with sleep-wake cycling without generalized epileptic activity was demonstrated (Fig. 1a).

On day 2, metabolic investigation revealed elevated levels of L-sulfocysteine, hypoxanthine, and xanthine, in combination with decreased uric acid in both plasma and urine, suggesting Moco deficiency. Clinical symptoms persisted, and the aEEG pattern changed from a continuous normal voltage pattern, via a discontinuous normal pattern into a burst suppression pattern. As the aEEG should be used as a complement to a standard electroencephalogram (EEG), a standard EEG was performed on day 2. It showed a burst suppression pattern with multifocal abnormalities, but no signs of epileptic activity at that moment (Fig. 1e). The cranial ultrasound showed a high echogenic signal in the white matter, representing white matter edema.

On day 3, the patient developed tonic-clonic seizures, and the aEEG showed electrographic correlation of repetitive seizure activity (Fig. 1b). Because of respiratory insufficiency, he was intubated and mechanically ventilated. Although treatment with phenobarbitone as antiepileptic drug was initiated, seizures continued. After the administration of midazolam (Fig. 1b, c), lidocaine, and pyridoxine, epileptic activity was suppressed. A flat trace pattern on the aEEG was registered (Fig. 1d). Magnetic resonance imaging (MRI) at day 3 showed marked white matter abnormalities and basal ganglia signal changes (Fig. 2).

From day 5 onwards, vigabatrin was started as an attempt for maintenance therapy. To reduce excessive sulfite levels, cysteamine was administered. Midazolam was diminished and subsequently stopped. The aEEG 
Fig. 1 Raw signal EEG traces (top two rows in a-d) and aEEG traces (bottom two rows in a-d) of neonate with Moco deficiency. a Day 1. Continuous normal voltage pattern with sleep-wake cycling without any seizures. $\mathbf{b}$ Day 3. Early morning, discontinuous pattern with repetitive seizures (arrows indicate administration of phenobarbitone and midazolam). c Day 3, morning. Not dense burst suppression pattern with repetitive seizure activity. d Day 3, evening. Flat trace EEG. e Standard EEG performed on day 2. Burst suppression pattern with multifocal abnormalities. No signs of epileptic activity
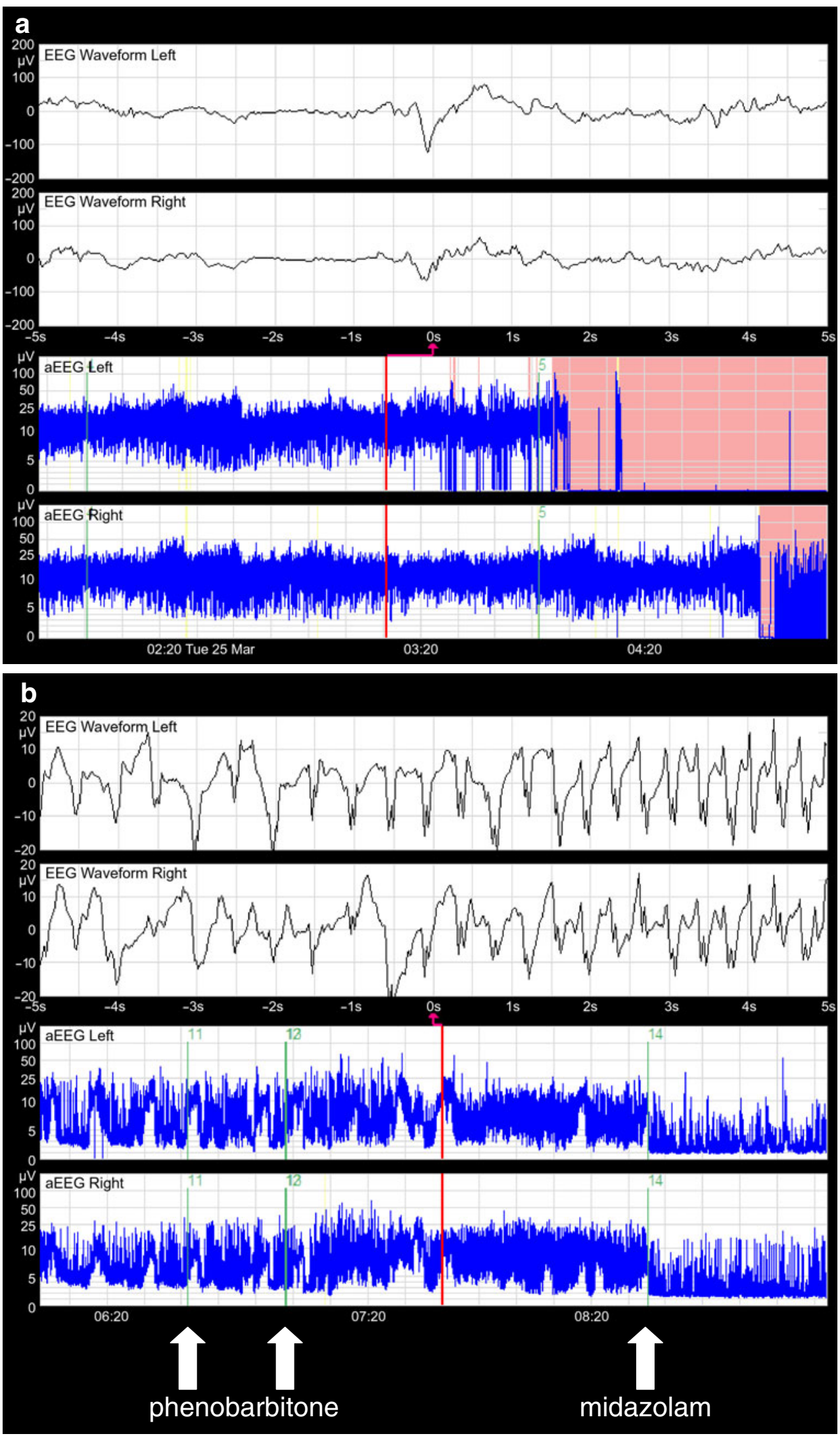


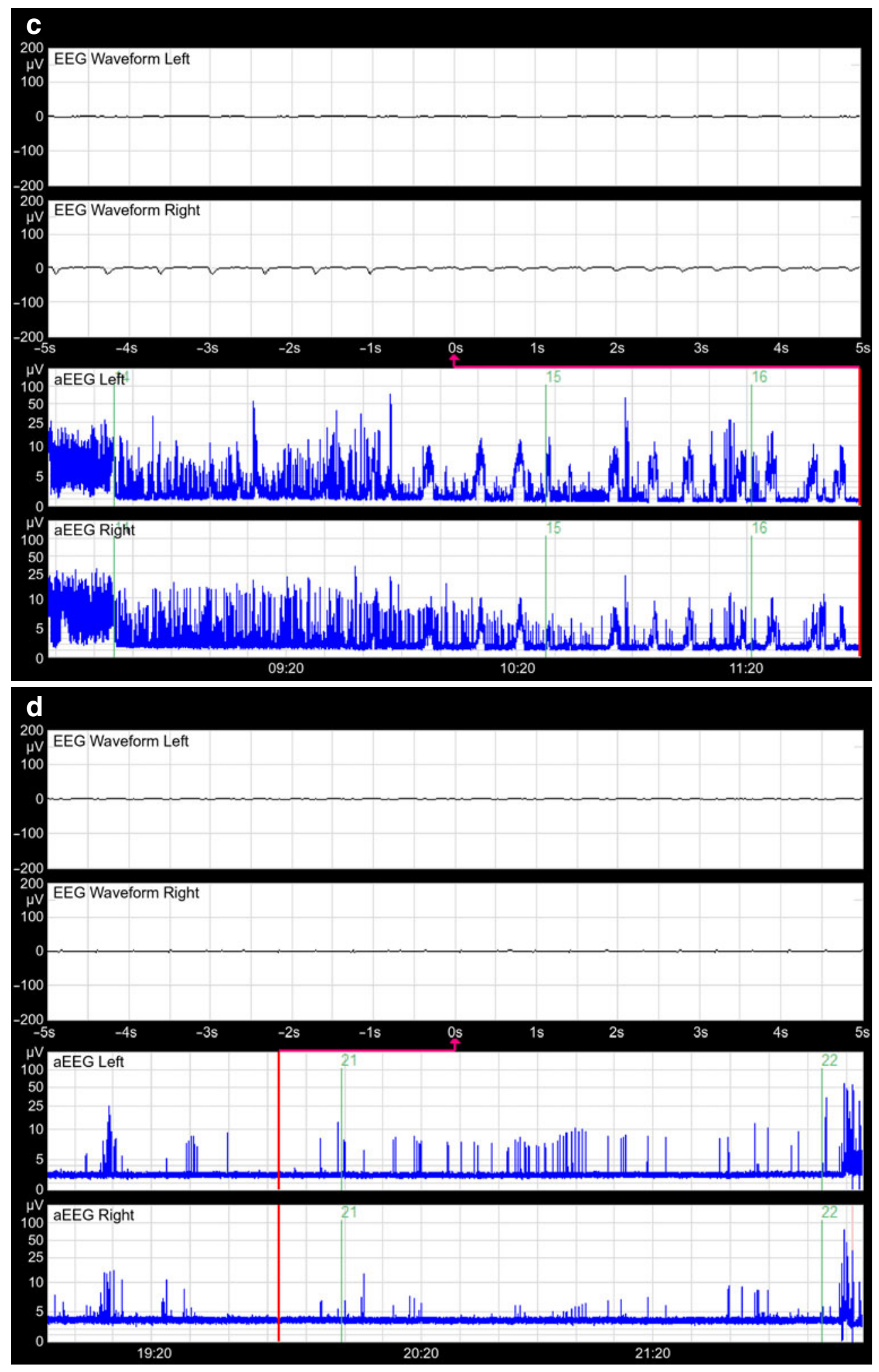

Fig. 1 (continued) 


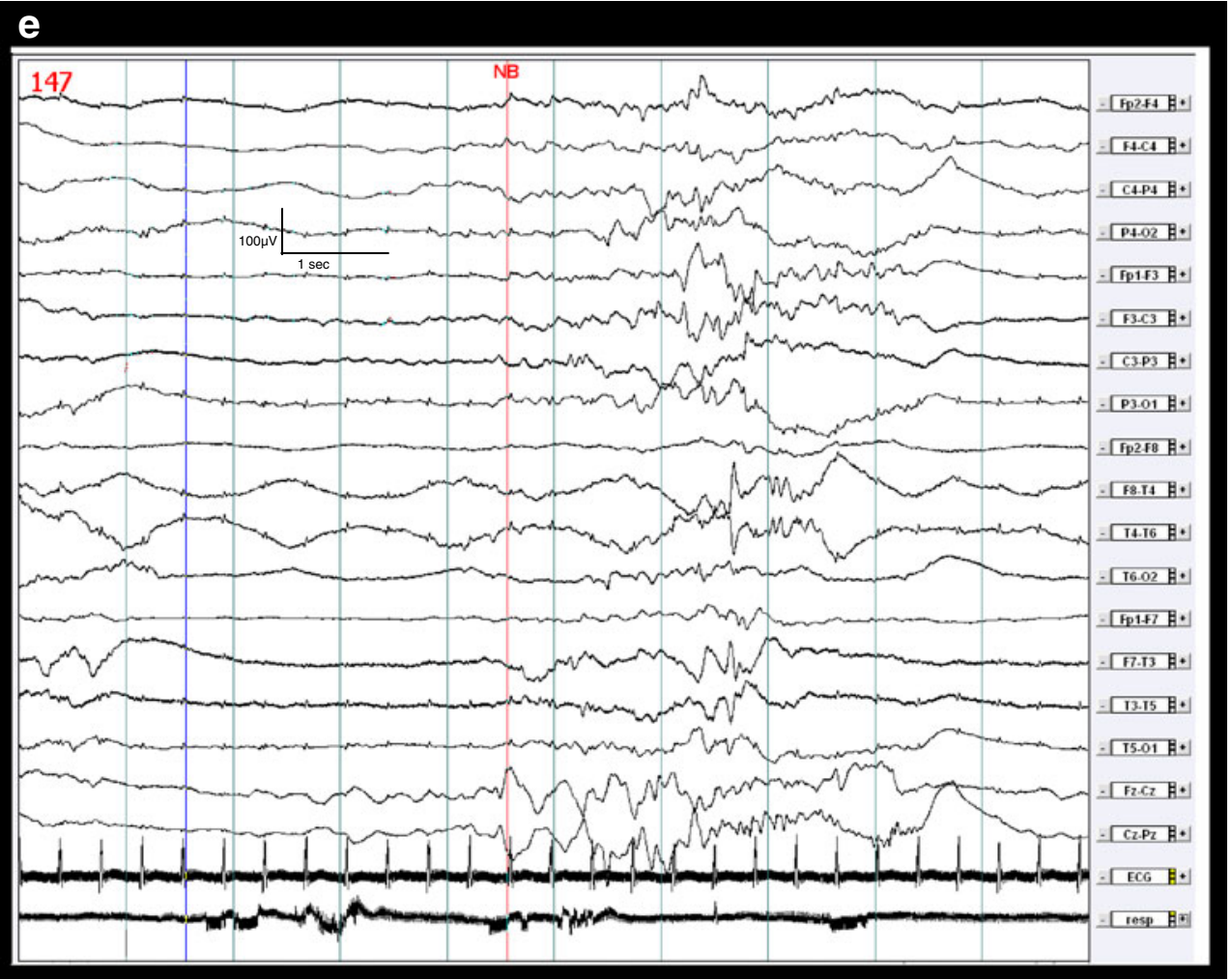

Fig. 1 (continued)

showed a continuous low voltage pattern with sporadic seizures. On day 11, the infant was weaned from mechanical ventilation. A few hours after detubation, the infant suffered from sudden unexplained respiratory and circulatory insufficiency, and resuscitation was started. However, despite maximum resuscitation, bradycardia persisted and the patient deceased. The parents did not give their consent for complete autopsy.

In our patient, the genotype analysis for Moco deficiency showed homozygosity for the novel mutation c.220C $>\mathrm{T}$ in exon 3 of the MOCS2 gene. Both parents are heterozygous carriers of this mutation. This mutation is located in the overlapping region of the two open reading frames in the MOCS 2 gene. In the 3 ' reading frame for the large subunit of molybdopterin synthase the identified mutation is silent. In the $5^{\prime}$ reading frame, however, the creation of a stop codon abolishes the highly conserved double glycin terminus of the small subunit. The identification of this clearly pathogenic mutation in the MOCS2 gene confirms the diagnosis of a Moco deficiency type B.

\section{Discussion}

In this case report, we describe the chronological changes in the aEEG in a patient with Moco deficiency type B.
He presented with signs of hypertonicity and myoclonic spasms shortly after birth. The aEEG at birth showed a normal background pattern with sleep-wake cycling without generalized epileptic activity. During the second day of life the aEEG changed from a continuous normal voltage pattern, via a discontinuous normal voltage pattern into a burst suppression pattern, possibly reflecting the progressive brain damage resulting from toxic sulfite accumulation. On the third day, aEEG showed status epilepticus, and after treatment a continuous low voltage registration with sporadic single seizures persisted. After discontinuation of midazolam, background pattern did not recover, reflecting severe brain damage (van Leuven et al. 2004).

The aEEG is based on a time-compressed semilogarithmic display of the peak-to-peak amplitude values of a filtered and rectified EEG signal. The number of channels differs between monitors. Hellstrom-Westas and Rosen (2006) suggested a new classification system for aEEG tracings suiting both term and preterm infants, describing three categories: background pattern (continuous, discontinuous, burst suppression, low voltage, or inactive/flat), sleep-wake cycling (absent, immature, or developed), and seizures. The aEEG can be used for the detection of seizures and evaluation of antiepileptic treatment. However, focal seizures or seizures of short 

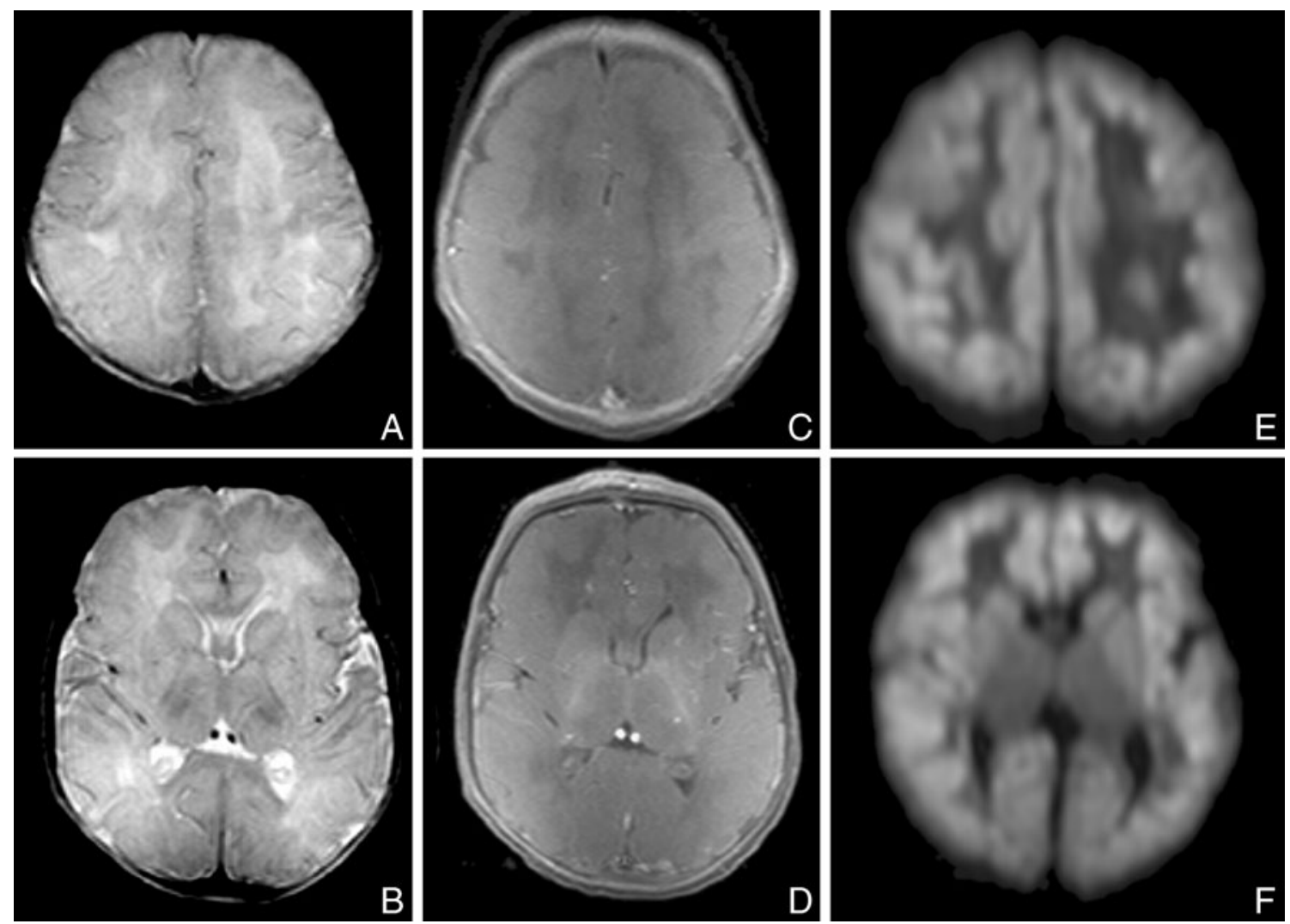

Fig. 2 T2-weighted (TR 3,000 ms, TE $120 \mathrm{~ms}$; a and b), and T1weighted (TR $480 \mathrm{~ms}$, TE $14 \mathrm{~ms}$; c and d) images of Moco-deficient neonate on 3rd day of life, showing loss of grey to white matter differentiation and changes in signal intensities in the basal ganglia.

duration may be missed with aEEG due to the reduced number of electrodes (Toet et al. 2002). Therefore, it should always be used as a complement to the standard EEG.

Moco deficiency is a rare autosomal-recessive inherited disorder leading to a combined deficiency of sulfite oxidase, xanthine dehydrogenase, and aldehyde oxidase. Absence of sulfite oxidase leads to sulfite accumulation and toxicity, causing severe neurologic impairment. Most affected infants are born after an uneventful pregnancy and delivery. More than $90 \%$ develop symptoms after several days; the others suffer from late onset disease after the neonatal period. Clinical symptoms include seizures refractive to therapy, myoclonic spasms, axial hypotonia, hypertonicity, and feeding difficulties (Johnson and Duran 2001).
Furthermore, extensive restricted diffusion is seen in the subcortical white matter and the cortex on the diffusion-weighted images (TR $3,400 \mathrm{~ms}$, TE $122 \mathrm{~ms}, \mathrm{~b} 1,000 \mathrm{~s} / \mathrm{mm}^{2}$; e and f)

Laboratory investigations usually reveal decreased serum levels of uric acid and increased excretion of sulfite, thiosulfate, S-sulfocysteine, taurine, hypoxanthine, and xanthine in urine. Cranial ultrasound imaging and MRI generally show subcortical cystic formation and atrophy (Serrano et al. 2007; Teksam et al. 2005).

Moco is synthesized in a multi-step biosynthetic pathway (Fig. 3). Four human genes are required for this pathway (MOCS1, MOCS2, MOCS3, and GEPH). Most pathogenic mutations have been identified in MOCS1 and MOCS2 (Reiss et al. 2005). Figure 3 shows a schematic presentation of human Moco biosynthesis, the involved gene products, and classification of Moco deficiency (Schwarz et al. 2009). Sulfite oxidase activity can be assayed in chorionic villus sampling tissue for use in
Type A

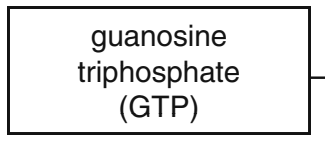

$\underset{\text { MOCS1 }}{\longrightarrow} \begin{gathered}\text { cyclic pyranopterin } \\ \text { monophosphate } \\ \text { (cPMP) }\end{gathered}$

Type B

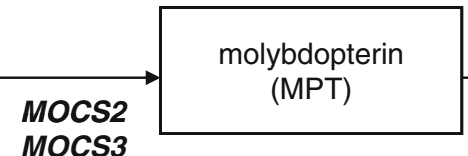

Type C

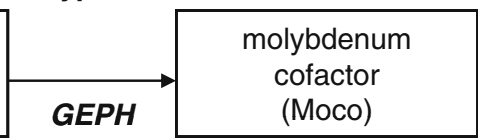

Fig. 3 Schematic pathway of Moco biosynthesis. Based on genetic and biochemical analysis, three types of Moco deficiency can be distinguished (Schwarz et al. 2009) 
prenatal diagnosis. Gene-based prenatal diagnosis is more informative than metabolite assays but should only be used in families in whom the affected gene and specific defect have been characterized (Johnson 2003).

Several attempts have been made to reduce sulfite production by dietary intervention or to absorb excessive sulfite with, for example, cysteamine administration. Seizures are often therapy resistant, however, with the introduction of novel antiepileptic drugs such as vigabatrin, seizure control has been observed in some patients (Johnson and Duran 2001). In 2004 Schwarz et al. described that repeated injections with cyclic pyranopterin monophosphate (cPMP, previously identified as precursor Z) into MOCS1-deficient mice resulted in extension of life span and normal development of these mice (Schwarz et al. 2004; Schwarz et al. 2009). Recently, the first patient with Moco deficiency type A was successfully treated according to this scheme (Veldman et al. 2010).

Until now, limited data have been available about the time profile in which neurological deterioration starts in patients with Moco deficiency. In a MOCS1-deficient mouse model it was demonstrated that these animals were indistinguishable from healthy littermates at birth, suggesting a maternal compensation for affected embryos in utero (Reiss et al. 2005). However, postnatally they fail to thrive and die within the first 12 days after birth, with an average life span of 7.5 days. Mice substituted with cPMP reached adulthood and had normal fertility, but withdrawal of cPMP from these MOCS1-deficient mice caused death within 10 14 days (Schwarz et al. 2004). Serrano et al. (2007) described chronological changes of cranial ultrasound images in a full term boy with Moco deficiency. Early cranial ultrasound showed nonspecific brain edema at day 3 and 5 , followed by white matter signal changes and cortical reduction at day 12 .

To our knowledge, this is the first case report describing the chronological changes in the aEEG in a patient with Moco deficiency type B. Although MRI and routine EEG were not performed at birth, significant brain damage at day 1 seems unlikely, as reflected by the normal aEEG pattern and normal cranial ultrasound findings at birth (Wezel-Meijler et al. 2010). In this patient, it is conceivable that maternal clearance of the neurotoxic metabolites was sufficient to protect the brain from severe injury. However, in the absence of such maternal clearance, there is accumulation of sulfite which in turn leads to permanent brain injury as shown by the progressive deterioration of the aEEG pattern. With the possibility of gene-based prenatal diagnosis of Moco deficiency and a possible treatment with injections of cPMP (for type A), insight into the time profile in which neurologic deterioration in Moco-deficient humans occurs or can be prohibited is essential.

Acknowledgements We thank the Department of Clinical Neurophysiology of the VU University Medical Center for providing the EEG.

Open Access This article is distributed under the terms of the Creative Commons Attribution Noncommercial License which permits any noncommercial use, distribution, and reproduction in any medium, provided the original author(s) and source are credited.

\section{References}

Hellstrom-Westas L, Rosen I (2006) Continuous brain-function monitoring: state of the art in clinical practice. Semin Fetal Neonatal Med 11:503-511

Johnson JL (2003) Prenatal diagnosis of molybdenum cofactor deficiency and isolated sulfite oxidase deficiency. Prenat Diagn 23:6-8

Johnson JL, Duran M (2001) Molybdenum cofactor deficiency and isolated sulfite oxidase deficiency. In: Scriver CR, Beander AL, Valle D, Sly WS (eds) The metabolic \& molecular bases of inherited disease. McGraw-Hill, New York, pp 3163-3177

Reiss J, Johnson JL (2003) Mutations in the molybdenum cofactor biosynthetic genes MOCS1, MOCS2, and GEPH. Hum Mutat 21:569-576

Reiss J, Bonin M, Schwegler H et al. (2005) The pathogenesis of molybdenum cofactor deficiency, its delay by maternal clearance, and its expression pattern in microarray analysis. Mol Genet Metab 85:12-20

Schwarz G, Santamaria-Araujo JA, WolfS et al. (2004) Rescue of lethal molybdenum cofactor deficiency by a biosynthetic precursor from Escherichiacoli.HumMolGenet 13:1249-1255

Schwarz G, Mendel RR, Ribbe MW (2009) Molybdenum cofactors, enzymes and pathways. Nature 460:839-847

Serrano M, Lizarraga I, Reiss J et al. (2007) Cranial ultrasound and chronological changes in molybdenum cofactor deficiency. Pediatr Radiol 37:1043-1046

Teksam O, Yurdakok M, Coskun T (2005) Molybdenum cofactor deficiency presenting with severe metabolic acidosis and intracranial hemorrhage. J Child Neurol 20:155-157

Toet MC, van der Meij W, de Vries LS, Uiterwaal CS, van Huffelen KC (2002) Comparison between simultaneously recorded amplitude integrated electroencephalogram (cerebral function monitor) and standard electroencephalogram in neonates. Pediatrics 109:772-779

van Leuven K, Groenendaal F, Toet MC et al. (2004) Midazolam and amplitude-integrated EEG in asphyxiated full-term neonates. Acta Paediatr 93:1221-1227

Veldman A, Santamaria-Araujo JA, Sollazzo S et al. (2010) Successful treatment of molybdenum cofactor deficiency type A with cPMP. Pediatrics 125:e1249-e1254

Wezel-Meijler G, Steggerda SJ, Leijser LM (2010) Cranial ultrasonography in neonates: role and limitations. Semin Perinatol 34:28-38 